\title{
Wave vector analysis methods using multi-point measurements
}

\author{
Y. Narita ${ }^{1}$, K.-H. Glassmeier ${ }^{1,2}$, and U. Motschmann ${ }^{3,4}$ \\ ${ }^{1}$ Institut für Geophysik und extraterrestrische Physik, Technische Universität Braunschweig, Mendelssohnstr. 3, \\ 38106 Braunschweig, Germany \\ ${ }^{2}$ Max-Planck-Institut für Sonnensystemforschung, Max-Planck-Str. 2, 37191 Katlenburg-Lindau, Germany \\ ${ }^{3}$ Institut für Theoretische Physik, Technische Universität Braunschweig, Mendelssohnstr. 3, 38106 Braunschweig, Germany \\ ${ }^{4}$ DLR-Institut für Planetenforschung, Rutherfordstr. 2, 12489 Berlin, Germany
}

Received: 24 June 2010 - Revised: 10 August 2010 - Accepted: 12 August 2010 - Published: 1 September 2010

\begin{abstract}
Recent developments of multi-point measurements in space provide a means to analyze spacecraft data directly in the wave vector domain. For turbulence study this means that we are able to estimate energy, helicity, and higher order moments in the wave vector domain without assuming Taylor's hypothesis or axisymmetry around the mean magnetic field. The methods of the wave vector analysis are presented and applied to four-point data of Cluster in the solar wind.
\end{abstract}

\section{Introduction}

Waves and turbulence observed in the interplanetary space and the Earth's magnetosphere are one of the most interesting subjects in space physics, as plasmas allow various kinds of linear wave modes as excitation states to exist (that are well documented by, e.g., Stix, 1992 and Gary, 1993) and also various kinds of nonlinear waves and turbulent states (Biskamp, 2003). One of the complexities in plasma turbulence is that not only eddy splitting but also Alfvén waves and other wave modes may be carriers of the energy cascade through wave-wave interactions. Earlier spacecraft observations in 1960s revealed that magnetic field fluctuations in the solar wind are reminiscent of turbulence, as their frequency spectra often exhibited a power-law spectrum characterized by the spectral index $-5 / 3$, the index known as Kolmogorov's inertial-range spectrum for hydrodynamic turbulence. Regions upstream and downstream of Earth's bow shock are also characterized by large-amplitude fluctuations, and they are thought to be in a turbulent state, too.

Correspondence to: Y. Narita (y.narita@tu-bs.de)
Earlier in-situ observations of space plasma turbulence were primarily limited to analyzing time series data based on single-spacecraft measurements, and the properties of the fluctuating magnetic field and flow velocity were determined in the temporal or frequency domain. Investigation of spatial properties of the fluctuating fields relied on Taylor's frozen-in flow hypothesis that neglects wave frequencies in the flow-rest frame when a fluctuating field is sampled in a fast-streaming medium (Taylor, 1938). This hypothesis relates the observed frequency with the wave number in the flow direction using the Doppler shift, $\omega \simeq \boldsymbol{k} \cdot \boldsymbol{V}$, where $\omega$ is the spacecraft-frame frequency, and $\boldsymbol{k}$ and $\boldsymbol{V}$ are the wave vector and the flow velocity vector, respectively. The solar wind is a fast-streaming plasma with the Mach number being 8 to 10 with respect to the sound speed and also the Alfvén speed. Therefore, Taylor's hypothesis is believed to be valid and has been widely applied in studying solar wind turbulence (Coleman, 1968; Matthaeus and Goldstein, 1982; Marsch and Tu, 1990; Podesta et al., 2007). Other physical quantities relevant to plasma turbulence such as the magnetic helicity density, the cross helicity, and the Elsässer variable spectra were also determined by adapting and reducing these quantities to the context of single-spacecraft measurements (Matthaeus and Goldstein, 1982; Matthaeus et al., 1982; Glassmeier et al., 1989; Marsch, 1991; Tu and Marsch, 1995).

It should be noted here that some fundamental properties of waves and turbulent fields are still missing because of the limitations of single-point measurements. For example, wave propagation speeds and directions, frequencies in the flow rest frame (that needs Doppler correction), and wavelengths cannot unambiguously determined in single-point measurements, otherwise one has to assume a wave mode or a dispersion relation to estimate these quantities. Separation between temporal and spatial variation in space plasma could not be

Published by Copernicus Publications on behalf of the European Geosciences Union and the American Geophysical Union. 
achieved in early single-point measurements because the Doppler shift was not corrected. Spatial properties of the fluctuating fields can be determined only by multi-point measurements: They allow us to determine the wave vectors, the wave propagation speeds and directions, the rest-frame frequencies by correcting the Doppler shift, and the fluctuation amplitudes associated with the wave vectors and the restframe frequencies. Furthermore, multi-point measurements also provide the opportunity to verify Taylor's hypothesis.

This paper reviews the wave vector analysis methods developed particularly for the Cluster mission (Escoubet et al., 2001; Balogh et al., 2001) to make use of full potential of the four-point measurements in space. Examples include (1) the wave telescope technique, (2) the extended wave telescope technique, and (3) the eigenvector analysis methods. The mathematical foundation of these methods are explained as well as application to the Cluster data. The wave telescope technique performs a parametric projection of the measured fluctuations into the wave vector domain and does not require any knowledge on dispersion relations nor Taylor's hypothesis. Using this technique and its extended methods, the distributions of energy and helicity can be determined in the frequency-wave vector domain. From the 3-D energy distribution, Taylor's hypothesis is verified for the first time for solar wind turbulence. Also, the analysis of bispectrum can be performed in the wave vector domain using the wave telescope technique. The concept of bispectrum represents a triple correlation that measures occurrence or strength of wave resonance processes among three different wave components, and therefore it serves as a useful analysis tool to investigate energy cascade process in turbulence. Details about the bispectrum analysis are explained in Sect. 3.3. The eigenvector analysis is another approach in wave analysis and provides methods to determine dispersion relations and highresolution wave number spectra.

\section{Projection methods}

It is of course ideal to have as many properly positioned spacecraft available as possible to Fourier transform observed fluctuations from the spatial coordinates into the wave numbers. The four measurement points of Cluster, from this point of view, are too few for performing a Fourier transform into the wave number domain, but it was proposed to estimate the energy distribution in the 4-D frequency-wave vector domain using four point measurements only. The idea to use multiple spacecraft as a plasma wave array experiment was originally raised by Musmann et al. (1974) before the concept of the Cluster mission was developed. The idea of the array experiment using multi-spacecraft was further developed for the Cluster mission (Neubauer and Glassmeier, 1990; Pinçon and Lefeuvre, 1991; Motschmann et al., 1996; Glassmeier et al., 2001), applying the projection methods earlier used in seismic wave studies (Capon, 1969; Harjes and Henger, 1973).

We define the state vector for an $L$-point measurements as

$\boldsymbol{S}(\omega)=\left(\begin{array}{c}S\left(\omega, \boldsymbol{r}_{1}\right) \\ S\left(\omega, \boldsymbol{r}_{2}\right) \\ \vdots \\ S\left(\omega, \boldsymbol{r}_{L}\right)\end{array}\right)$.

Here each sensor measures a scalar quantity $S$ at the $i$-th position of the sensors $\boldsymbol{r}_{i}$. The field $S$ is already transformed from the time into the frequency domain, and is a function of the frequency $\omega$ and the position $\boldsymbol{r}_{i}$. Consider a projection of the state vector, which provides the amplitude as a function of the frequency and the wave vector. In other words, the state vector is reduced to a scalar by taking a dot product with a suitable weight or projection vector. We measure a scalar field using a sensor-array and determine the state vector in the frequency domain; and then the state vector is reduced to a scalar. The projected quantity is the wave amplitude given as a complex number, retaining the phase information in the frequency-wave vector domain:

$S(\omega, \boldsymbol{k})=\boldsymbol{w}^{\dagger}(\omega, \boldsymbol{k}) \cdot \boldsymbol{S}(\omega)$,

where $\boldsymbol{w}^{\dagger}(\omega, \boldsymbol{k})$ denotes the weight vector or the projection vector (the dagger means Hermitian conjugate). One may also estimate the wave power in the frequency-wave vector domain as:

$P(\omega, \boldsymbol{k})=\left\langle|S(\omega, \boldsymbol{k})|^{2}\right\rangle=\boldsymbol{w}^{\dagger}(\omega, \boldsymbol{k}) \mathbf{R}(\omega) \boldsymbol{w}(\omega, \boldsymbol{k})$,

where $\mathbf{R}(\omega)$ denotes the $L$-by- $L$ cross spectral density (CSD) matrix determined by the state vector:

$\mathbf{R}(\omega)=\frac{1}{T}\left\langle\boldsymbol{S}(\omega) \boldsymbol{S}^{\dagger}(\omega)\right\rangle$.

The factor $1 / T$ represents a division by the measured time length $T$, so that the elements of the CSD matrix are given as energy density in the frequency domain, in units of squared amplitude per frequency. The angular bracket $\langle\cdots\rangle$ represents the operation of averaging. We use in our data analysis the time averaging, assuming that fluctuations are stationary. The task is now to find the weight vector in a suitable form describing the amplitude or the power associated with the parameter $\boldsymbol{k}$, the wave vector. We introduce two different projection methods: beam-former projection and Capon's minimum variance projection.

\subsection{Beam-former projection}

Let us define the steering vector that describes a plane wave approximation characterized by the wave vector $\boldsymbol{k}$ :

$\boldsymbol{h}(\boldsymbol{k})=\left(\begin{array}{c}\mathrm{e}^{i \boldsymbol{k} \cdot \boldsymbol{r}_{1}} \\ \mathrm{e}^{i \boldsymbol{k} \cdot \boldsymbol{r}_{2}} \\ \vdots \\ \mathrm{e}^{i \boldsymbol{k} \cdot \boldsymbol{r}_{L}}\end{array}\right)$. 
The beam-former projection uses the steering vector as the weight vector,

$\boldsymbol{w}=\boldsymbol{h}$,

and the wave power is estimated as

$P_{\mathrm{BF}}=\boldsymbol{h}^{\dagger} \mathbf{R} \boldsymbol{h}$.

An example of the wave number spectrum evaluated with the beam-former projection technique is displayed in Fig. 1 for synthetic data. In this example, a single plane wave is generated with the wave number $k=0.021 \mathrm{rad} / \mathrm{km}$ and the fluctuating field is sampled at four distinct points in a 1-D array as time series data. The evaluated spectrum shows a maximum at the signal wave number, but the spectrum is very broad and flat and identification of the signal wave number under the high background level in the spectrum is difficult. Furthermore, the spectrum exhibits a drop at relatively small wave numbers. The result is therefore much different from the spectrum of the synthetic data.

\subsection{Capon's minimum variance projection}

To reduce the high background level of the beam-former spectrum and to make the spectrum have a sharp peak at the signal wave vector, Capon (1969) proposed the method of minimum variance projection. Consider minimizing the power while keeping the fluctuation amplitude at the looking wave vector $\boldsymbol{k}$ unchanged (referred to as the unit gain constraint). The task is to minimize the interference in the spectrum that come from wave vectors other than the looking wave vector. This problem can be formulated as an optimization problem under a constraint as follows:

minimize $\quad \boldsymbol{w}^{\dagger} \mathbf{R} \boldsymbol{w} \quad$ subject to $\quad \boldsymbol{w}^{\dagger} \cdot \boldsymbol{h}=1$

or

$\delta\left[\boldsymbol{w}^{\dagger} \mathbf{R} \boldsymbol{w}-\lambda\left(\boldsymbol{w}^{\dagger} \cdot \boldsymbol{h}-1\right)\right]=0$,

with the Lagrangian multiplier $\lambda$. Capon (1969) obtained the analytical solution for this problem as follows:

$\boldsymbol{w}=\frac{\mathbf{R}^{-1} \boldsymbol{h}}{\boldsymbol{h}^{\dagger} \mathbf{R}^{-1} \boldsymbol{h}}$.

The projected power is therefore

$P_{\mathrm{C}}=\frac{1}{\boldsymbol{h}^{\dagger} \mathbf{R}^{-1} \boldsymbol{h}}$.

See, for example, Haykin (1991) for the derivation. It is worthwhile to note that Capon's projection vector is determined not only by the steering vector but also by the measurement itself through the state vector. An example of the spectrum evaluated by Capon's method is presented in Fig. 1 for the same data as that used for the beam-former spectrum. The Capon spectrum shows a much clearer peak at the signal wave number and the background level is significantly reduced.

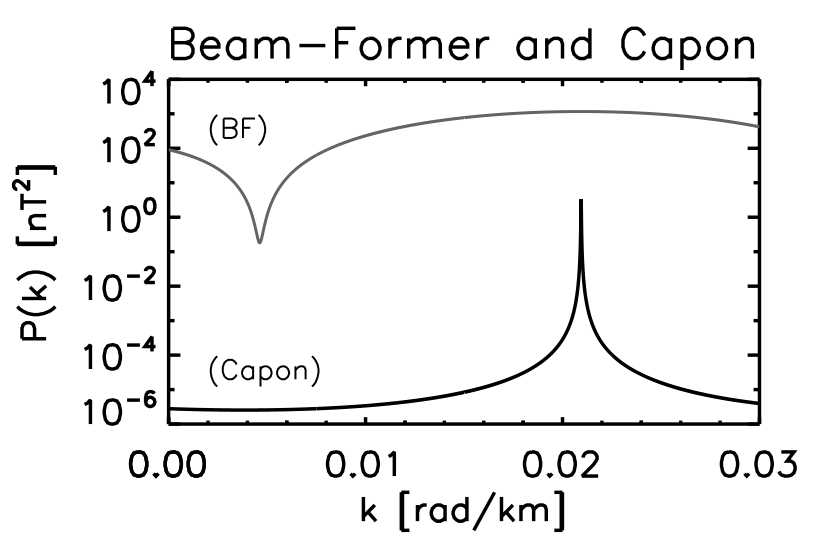

Fig. 1. Wave number spectra evaluated by the beam-former (BF) and Capon's projection methods.

\subsection{Wave Telescope Technique}

The projection method can be generalized to measurements of vectors such as the magnetic field, and the state vector has $3 L$ elements (3 components of the vector measured by $L$ sensors). In the case of the Cluster magnetometer data the number of sensor is $L=4$, and the state vector is established as:

$\boldsymbol{S}(\omega)=\left(\begin{array}{c}\boldsymbol{B}_{1}(\omega) \\ \boldsymbol{B}_{2}(\omega) \\ \vdots \\ \boldsymbol{B}_{4}(\omega)\end{array}\right)$,

and the generalized CSD matrix is

$\mathbf{R}(\omega)=\frac{1}{T}\left\langle\boldsymbol{S}(\omega) \boldsymbol{S}^{\dagger}(\omega)\right\rangle$.

The CSD matrix is a $3 L \times 3 L$ matrix and depends on the frequency. After the projection, the CSD matrix is reduced to a $3 \times 3$ matrix, and each element of the matrix represent the correlation among the $x, y$, and $z$ component of the fluctuating magnetic field as a function of the frequency and the wave vector. The steering vector is a $3 L \times 3$ matrix:

$\mathbf{H}(\boldsymbol{k})=\left(\begin{array}{c}\mathbf{I} \mathrm{e}^{i \boldsymbol{k} \cdot \boldsymbol{r}_{1}} \\ \mathbf{I} \mathrm{e}^{i \boldsymbol{k} \cdot \boldsymbol{r}_{2}} \\ \mathbf{I} \mathrm{e}^{i \boldsymbol{k} \cdot \boldsymbol{r}_{3}} \\ \mathbf{I} \mathrm{e}^{i \boldsymbol{k} \cdot \boldsymbol{r}_{4}}\end{array}\right)$,

where I denotes the $3 \times 3$ unit matrix. The formula of Capon's projection can be generalized to matrix operations, and we obtain the projection matrix as (Pinçon and Lefeuvre, 1991; Motschmann et al., 1996)

$\mathbf{W}=\mathbf{R}^{-1} \mathbf{H}\left[\mathbf{H}^{\dagger} \mathbf{R}^{-1} \mathbf{H}\right]^{-1}$

under the unit gain constraint:

$\mathbf{W}^{\dagger} \mathbf{H}=\mathbf{I}$. 
The projected matrix is expressed as:

$\mathbf{P}=\left[\mathbf{H}^{\dagger} \mathbf{R}^{-1} \mathbf{H}\right]^{-1}$.

This is a $3 \times 3$ correlation matrix in the frequency-wave vector domain. The diagonal and off-diagonal elements represent the fluctuation power and wave helicity, respectively. The trace of the matrix gives the total fluctuation power.

In addition, one may impose an additional constraint that the field satisfies the divergence-free condition (Pinçon and Lefeuvre, 1991; Motschmann et al., 1996). This can be expressed as

$\boldsymbol{k} \cdot \mathbf{W}^{\dagger} \boldsymbol{S}=0$.

This condition may be reflected to the state vector $\mathbf{S}$ such that $S$ is replaced by $\mathbf{V} \boldsymbol{S}$, where the matrix $\mathbf{V}$ represents a projection of the state vector onto the plane perpendicular to the wave vector:

$\mathbf{V}=\mathbf{I}+\frac{k \boldsymbol{k}}{k^{2}}$.

The matrix $\mathbf{V}$ can be directly incorporated in Capon's spectrum and we obtain the projection matrix as

$\mathbf{W}=\mathbf{R}^{-1} \mathbf{H V}\left[\mathbf{V}^{\dagger} \mathbf{H}^{\dagger} \mathbf{R}^{-1} \mathbf{H V}\right]^{-1}$

and the projected matrix:

$\mathbf{P}_{\mathrm{WT}}=\left[\mathbf{V}^{\dagger} \mathbf{H}^{\dagger} \mathbf{R}^{-1} \mathbf{H V}\right]^{-1}$.

The trace of the projected matrix gives the total fluctuation power at frequency $\omega$ and the wave vector $\mathbf{k}$ :

$P_{\mathrm{WT}}=\operatorname{tr}\left(\left[\mathbf{V}^{\dagger} \mathbf{H}^{\dagger} \mathbf{R}^{-1} \mathbf{H V}\right]^{-1}\right)$.

Estimating the power in the wave vector domain using the three matrices $\mathbf{R}, \mathbf{H}$, and $\mathbf{V}$ is called the wave telescope technique or k-filtering (Pinçon and Lefeuvre, 1991; Motschmann et al., 1996; Glassmeier et al., 2001; Pinçon and Motschmann, 1998; Pinçon and Glassmeier, 2008), and it provides the means to determine the energy distribution in the 4-D frequency-wave vector domain using Cluster data. Note that the projection vector $\boldsymbol{w}$ and the projection matrix $\mathbf{W}$ are the dimensionless operators and they do not change the units of the spectrum after the projection, i.e., the same dimension as the CSD matrix (squared amplitude per frequency). A suitable procedure is needed to properly evaluate Capon's spectra as energy distributions or energy density spectra. After integration of Capon's spectra over frequencies the spectra are given in units of power (squared amplitude), and the division by the wave number interval after the integration the spectra are adapted to the energy density spectra in units of squared amplitude per wave number.
An example of the 3-D energy distribution in the wave vector domain evaluated by the wave telescope technique is displayed in Fig. 2. In this example, synthetic time series data are generated from the model energy distribution (which is an isotropic distribution in the wave vector domain) with random phases. The fluctuations are sampled at four discrete points forming a tetrahedron. The energy distribution is then reconstructed from four-point time series data using the wave telescope technique. Although the reconstructed distribution is not exactly the same as the model distribution, the overall structure can be reasonably well reconstructed (Narita et al., 2010a). The wave telescope technique has the advantage that it does not require any knowledge on the number of signals. In the MUSIC algorithm (presented later), one has to know the number of signals. It is worthwhile to note that Capon's projection method is valid not only for plane waves but also for other spatial structures. Constantinescu et al. (2006, 2007) generalized the wave telescope technique for spherical wave patterns. Plaschke et al. (2008) generalized it to field-line-resonant phase patterns of ULF pulsations of the geomagnetic field using phase-shifted waves. The associated spatial patterns are displayed in Fig. 3.

The wave telescope technique was extensively tested using synthetic data set (Pinçon and Lefeuvre, 1991; Motschmann et al., 1996; Glassmeier et al., 2001; Narita et al., 2010a; Sahraoui et al., 2010), and limitations of the wave telescope technique have been discussed, too. From the viewpoint of calculation, the spectral estimator of the wave telescope technique works for arbitrary wave vectors as they are a parameter in the analysis. In practice, tetrahedral configuration imposes the upper and lower limits of the range of wave vectors. The upper limit of the wave number is determined by the spacecraft separation (Nyquist wave number), while the lower limit is determined by the cognition of large-scale wavelength. Sahraoui et al. (2010) argue that $1 / 50$ of the maximum or Nyquist wave number is a reasonable choice as the lower limit of the wave number: below this limit the uncertainty in the relation between the estimated power and the wave numbers is too large. The mean field should be uniform in the collected data for the wave telescope technique to be applicable (Glassmeier et al., 2001). The tetrahedral configuration should also be carefully checked when applying the wave telescope technique. The Nyquist wave number is, strictly speaking, dependent on the directions because the sensor separation distance changes when projected to various directions. The tetrahedral configuration of the sensors therefore imposes a particular window or cell in the wave vector domain where the wave telescope analysis is valid (known as the first Brillouin zone in solid state physics). It was discussed recently that the shape of the Brillouin may influence the shape of evaluated spectral distribution, known as the spatial aliasing problem (Narita and Glassmeier, 2009a; Sahraoui et al., 2010). This effect can be minimized when the tetrahedral configuration is regular. 

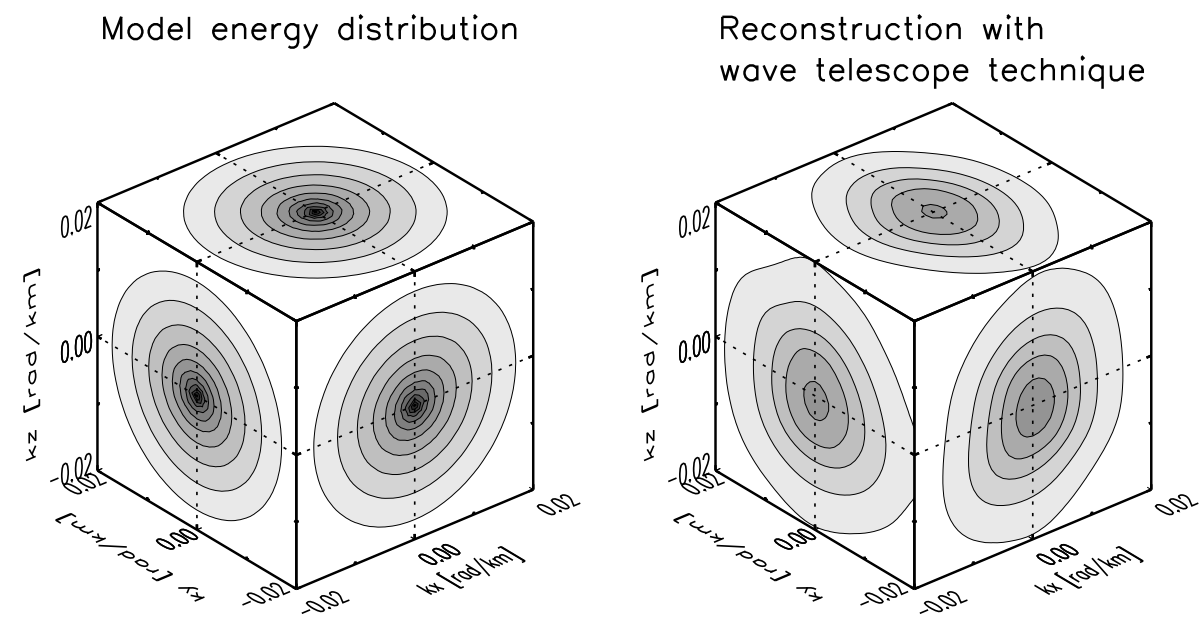

Fig. 2. Comparison of energy distribution in the 3-D wave vector domain. Left panel displays the model distribution from which synthetic data are generated and sampled at four different positions in the spatial coordinate. Right panels displays the energy distribution reconstructed using the wave telescope technique. Adapted from the numerical test of the wave telescope technique in Narita et al. (2010a).

As an application to the Cluster data, Fig. 4 displays the 3-D energy distribution in the wave vector domain for magnetic field fluctuations in the solar wind. The energy distribution is determined in the 4-D frequency-wave vector domain in the spacecraft frame, and then the Doppler shift is corrected and the distribution is transformed into the flow rest frame. Finally, the 4-D distribution is reduced to the 3-D distribution by integrating over the rest-frame frequencies. The distribution is anisotropic and exhibits an extended structure perpendicular to the mean magnetic field direction, suggesting that the observed solar wind fluctuations represent the geometry of quasi-2-D turbulence. While earlier measurements based on single-point measurements already revealed the anisotropy in solar wind turbulence, Taylor's hypothesis and axisymmetry around the mean field had to be assumed to infer the energy distribution in three-dimension (Matthaeus et al., 1990; Carbone et al., 1995; Dasso et al., 2005). The wave telescope technique does not require such assumptions and determines the 3-D energy distribution directly in the wave vector domain. One of the most important conclusions of the 3-D energy distribution is that the turbulence in solar wind is not axisymmetric about the background magnetic field (Narita et al., 2010c). This was not seen before the Cluster mission. Various interpretations are possible to explain the non-axisymmetric structure: it may originate in the coronal magnetic field structure, or perhaps it is caused by the radial expansion of solar wind. Statistical study of the 3-D energy distributions on various spatial scales would be a suitable task for this problem, which is being carried out currently.

It is furthermore possible to verify Taylor's hypothesis using the 3-D energy distribution. The 1-D energy spectrum is estimated by reducing the 3-D distribution into 1-D wave number domain in the flow direction and transforming it into the energy density in the wave number domain (in units of

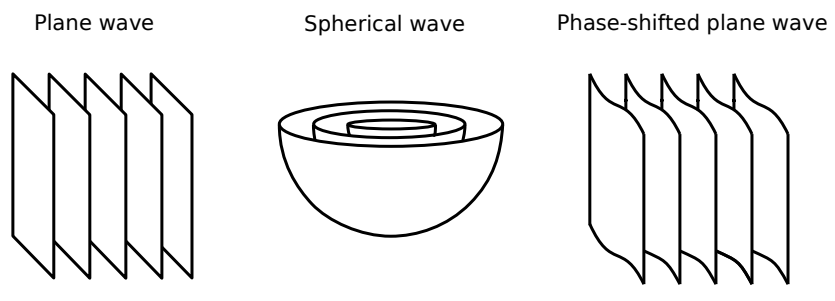

Fig. 3. Spatial patterns that can be used for the Capon's projection technique, after Constantinescu (2007).

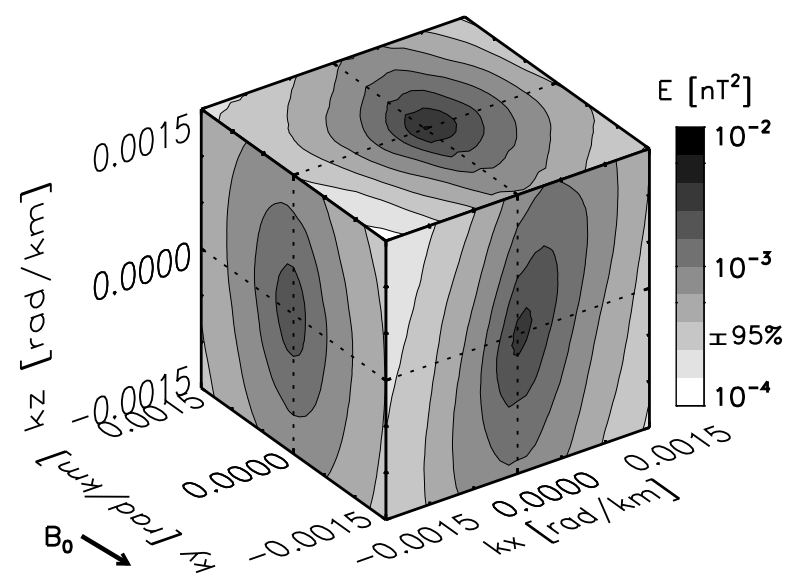

Fig. 4. Cubic representation of magnetic energy distribution in the 3-D wave vector domain for solar wind turbulence measured by Cluster. The cube shows the distribution in the GSE coordinate system. The 3-D distribution is projected onto three planes by averaging over the wave vector components perpendicular to the planes. The $95 \%$ confidence interval is displayed at the contour scale bar. Directions of the mean magnetic field $\mathbf{B}_{0}$ is indicated as well (Narita et al., 2010c). 
squared amplitude per wave number interval). The energy spectrum in the flow direction is then compared with that evaluated using Taylor's hypothesis in Fig. 5. The spectrum estimated from the wave telescope technique (upper panel) shows deviation from the power-law spectrum with the index $-5 / 3$ at larger wave numbers. The spectrum estimated from Taylor's hypothesis (lower panel) can be fitted on the whole by a power-law with the index $-5 / 3$, but the spectrum shows deviations (or fluctuations) from the power-law curve at various wave numbers. The direct comparison between the two spectra suggests that Taylor's hypothesis appears to be valid, but one should keep in mind that the resolution of the wave numbers is not very satisfactory in the reduced spectrum (upper panel), and further investigations would be needed to verify on what scales Taylor's hypothesis clearly breaks down. Taylor's hypothesis neglects wave frequencies in the flowrest frame compared to the Doppler term, the breakdown of Taylor's hypothesis is expected when the rest-frame frequencies cannot be neglected. Another possibility of the breakdown of Taylor's hypothesis is that there is no dispersion relation in the fluctuations and the energy distribution spreads both in the frequency and wave vector domain, such that higher frequencies in the flow-rest frame contribute significantly as well as lower frequencies. Therefore, whether or not a dispersion relation exists and how the energy spectrum in the rest-frame frequency domain looks like are very important questions in turbulence. The energy spectra in the wave vector domain are evaluated for magnetic field fluctuation in various regions: in the solar wind (Narita et al., 2010a,b,c), foreshock (Narita et al., 2006; Narita and Glassmeier, 2010d) and magnetosheath (Sahraoui et al., 2003, 2006; Narita and Glassmeier, 2010d).

\section{Extended wave telescope technique}

\subsection{Magnetic helicity density}

The wave telescope technique provides a $3 \times 3$ matrix with each element representing a correlation among $B_{x}, B_{y}$, and $B_{z}$ components of fluctuations in the frequency-wave vector domain. While the diagonal elements of the CSD matrix represent the wave power, the off-diagonal elements represent cross-correlations and contain information about the magnetic helicity density. To relate the projected CSD matrix with the magnetic helicity density, we use the expression of the vector potential $\boldsymbol{a}$ for the fluctuating magnetic field $\boldsymbol{b}$ :

$\boldsymbol{a}=-\frac{i}{k^{2}} \boldsymbol{k} \times \boldsymbol{b}$

The magnetic helicity density can be determined by building a scalar product between the vector potential $\boldsymbol{a}$ and the magnetic field $\boldsymbol{b}$ :

$h^{M}=\left\langle\boldsymbol{a}^{\dagger} \cdot \boldsymbol{b}\right\rangle$

where the angular bracket $\langle\cdots\rangle$ represents the averaging over different ensembles, e.g., averaging over different time se-
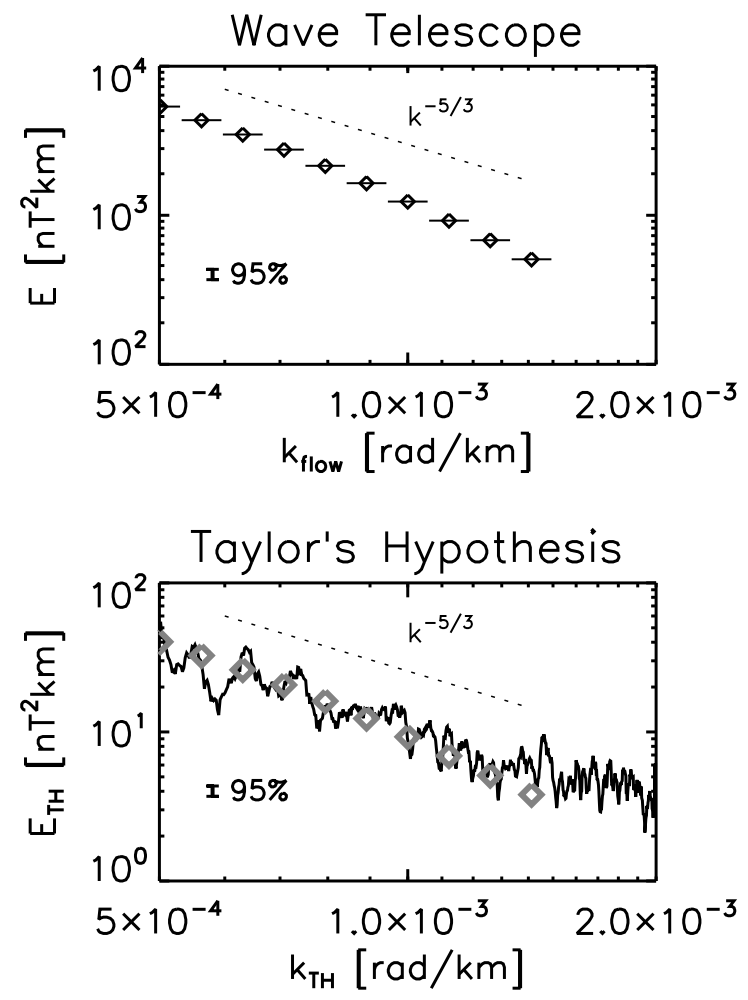

Fig. 5. Comparison of the wave number spectra. The upper panel is the energy spectrum for the wave number in the flow direction, reduced from the 3-D energy distribution using Cluster data and the wave telescope technique. The lower panel displays the energy spectrum transformed from the frequency into the wave number domain using Taylor's hypothesis (solid line) and the reduced spectrum shown in the upper panel (squares) with adjustment for comparison of the spectral slope.

ries data. The magnetic helicity density is related to the offdiagonal elements of the projected CSD matrix as (Narita et al., 2009b):

$h^{M}=-\frac{i}{k^{2}}\left[k_{x}\left(P_{y z}-P_{z y}\right)+k_{y}\left(P_{z x}-P_{x z}\right)+k_{z}\left(P_{x y}-P_{y x}\right)\right]$.

It is worthwhile to note that the estimate of the helicity density Eq. (24) is constructed as gauge-independent, and it is evaluated in the frequency-wave vector domain.

As an example, the distributions of the magnetic helicity density in the frequency-wave number domain along the mean magnetic field is displayed and compared with that of the fluctuation energy (Fig. 6). The data are taken from Cluster magnetometer data in the terrestrial foreshock region. The distributions are corrected for the Doppler shift. The helicity density can take both a positive and a negative value, and the magnitude of the helicity density is plotted here. Both the energy and the helicity density distributions exhibit several peaks almost at the same frequencies and the wave numbers. While the energy distribution shows wave activities for any 

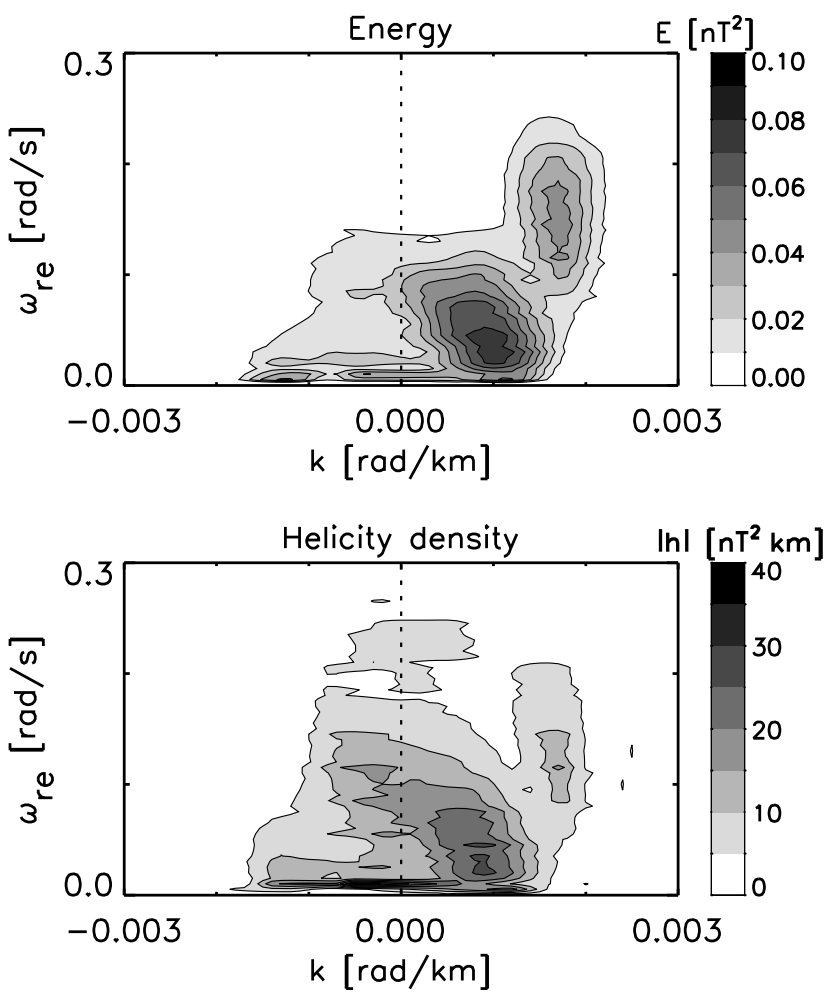

Fig. 6. Energy and helicity density in the frequency-wavenumber domain for a synthetic data set of four-point measurements of magnetic field (Narita et al., 2009b).

kind of polarization or helicity (i.e., linear, circular, and elliptical polarization), the helicity distribution shows the activities for the circular or elliptical sense of field rotation. The peaks are located on the positive wave number side, parallel to the mean magnetic field and it is also in the direction away from the shock to the interplanetary space. From the rest-frame frequencies and the wave numbers one may estimate the phase speeds, and they are about $v_{\mathrm{ph}}=40 \mathrm{~km} / \mathrm{s}$ and $v_{\mathrm{ph}}=88 \mathrm{~km} / \mathrm{s}$ at $k=0.0010 \mathrm{rad} / \mathrm{km}$ and $k=0.0017 \mathrm{rad} / \mathrm{km}$, respectively. The latter peak is close to the Alfvén speed of the background plasma, about $77 \mathrm{~km} / \mathrm{s}$, while the first one is about half of the Alfvén speed. Figure 6 suggests that the foreshock waves represent the growth of ion instabilities driven by ion beams moving sunward from the shock. The energy and magnetic helicity distributions in the frequencywave number domain essentially agree with the predictions based on the wave kinetic theory (Gary, 1993).

\subsection{Wave telescope for flow velocity data}

Although the wave telescope technique was developed particularly for analyzing multi-point magnetic field data, it can be applied to to the multi-point flow velocity data. For example, Cluster electron data are available at four spacecraft and the data is suitable for the wave telescope analysis. However, the divergence-free condition is not always valid for flow ve- locity fields and the wave telescope technique for the flow velocity should use the form of Eq. (16). In a similar fashion to the approach used in the magnetic helicity density, various quantities relevant to fluid turbulence may be evaluated using the flow velocity data. The kinetic energy is the trace of the projected CSD matrix, and the off-diagonal elements of the matrix provide the kinetic helicity density $h^{\mathrm{K}}=\langle\boldsymbol{u} \cdot \boldsymbol{\Omega}\rangle$. The symbol $\boldsymbol{\Omega}$ denotes the vorticity, the curl of the flow velocity. The kinetic helicity density is related to the projected CSD matrix as:

$$
\begin{aligned}
h^{\mathrm{K}}= & \left\langle\boldsymbol{u}^{\dagger} \cdot \boldsymbol{\Omega}\right\rangle \\
= & i\left[k_{x}\left(P_{u, y z}-P_{u, z y}\right)+k_{y}\left(P_{u, z x}-P_{u, x z}\right)\right. \\
& \left.+k_{z}\left(P_{u, x y}-P_{u, y x}\right)\right] .
\end{aligned}
$$

In this expression we used the vorticity in the Fourier domain:

$\boldsymbol{\Omega}=i \boldsymbol{k} \times \tilde{\boldsymbol{u}}$,

where the tilde-hat represents the Capon projection into the frequency-wave vector domain, and $P_{u, i j}$ denotes the the $(i, j)$-component of the projected CSD matrix for the flow velocity. It is also possible to evaluate the enstrophy (the squared vorticity) and the dilatation (the divergence of the velocity, which is a measure of the fluid compressibility). The former is given as

$\Omega^{2}=|\boldsymbol{k} \times \tilde{\boldsymbol{u}}|^{2}$,

and the latter is

$d^{2}=|\boldsymbol{k} \cdot \tilde{\boldsymbol{u}}|^{2}$,

Finally, the correlation between the flow velocity and the magnetic field gives the cross helicity density, $h^{\mathrm{C}}=\left\langle\tilde{\boldsymbol{u}}^{\dagger} \cdot \tilde{\boldsymbol{b}}\right\rangle$. This can be evaluated in the frequency-wave vector domain, too.

\subsection{Higher order moments}

Using the wave telescope technique it is possible to estimate higher order moments which are useful quantities to study wave-wave interactions. For example, third order moments (also named bispectra or three-point correlations) are the measure of three-wave couplings and can be determined in the frequency-wave vector domain. Three-wave processes are characterized by the resonance condition of frequencies and wave vectors, described as $\omega^{\prime \prime}=\omega \pm \omega^{\prime}$ and $\boldsymbol{k}^{\prime \prime}=\boldsymbol{k} \pm \boldsymbol{k}^{\prime}$. One of the third order moments relevant in plasma physics the triple correlation of two fluctuation components of the magnetic field and a density fluctuation component under the conditions $\omega^{\prime \prime}=\omega+\omega^{\prime}$ and $\boldsymbol{k}^{\prime \prime}=\boldsymbol{k}+\boldsymbol{k}^{\prime}$ :

$$
F\left(\omega, \omega^{\prime}, \boldsymbol{k}, \boldsymbol{k}^{\prime}\right)=\left\langle b(\omega, \boldsymbol{k}) n\left(\omega^{\prime}, \boldsymbol{k}^{\prime}\right) b^{*}\left(\omega+\omega^{\prime}, \boldsymbol{k}+\boldsymbol{k}^{\prime}\right)\right\rangle,
$$

where we used, for simplicity, the scalar fields of the magnetic field $b$ and number density $n$. These fields are 
projected into the frequency-wave number domain using Capon's method:

$$
\begin{aligned}
& b(\omega, \boldsymbol{k})=\boldsymbol{w}_{b}^{\dagger}(\omega, \boldsymbol{k}) \cdot \boldsymbol{S}_{b}(\omega) \\
& n(\omega, \boldsymbol{k})=\boldsymbol{w}_{n}^{\dagger}(\omega, \boldsymbol{k}) \cdot \boldsymbol{S}_{n}(\omega)
\end{aligned}
$$

Here $\boldsymbol{S}_{b}(\omega)$ and $\boldsymbol{S}_{n}(\omega)$ are the state vectors of the magnetic field and the number density, and $\boldsymbol{w}_{b}$ and $\boldsymbol{w}_{n}$ are the respective projection vectors. The asterisk denotes the operation of complex conjugate. The meaning of the bispectrum is as follows. If three waves are in resonance in the measured data, the bispectrum returns a non-zero quantity, otherwise the bispectrum for the other non-resonant waves returns a small value close to zero due to the averaging operation. The bispectrum can be investigated for the plus-sign coupling $\left(\omega+\omega^{\prime}\right.$ and $\left.\boldsymbol{k}+\boldsymbol{k}^{\prime}\right)$ and for the minus-sign coupling $\left(\omega-\omega^{\prime}\right.$ and $\left.\boldsymbol{k}-\boldsymbol{k}^{\prime}\right)$.

The bispectrum was evaluated using Cluster data in the wave vector domain for the coupling $\boldsymbol{k}_{b}+\boldsymbol{k}_{n}=\boldsymbol{k}_{b}^{\prime}$ (Fig. 7), where $\boldsymbol{k}_{b}$ and $\boldsymbol{k}_{n}$ denote the wave numbers of the fluctuating magnetic field and the electron density, respectively. The primed wave vector $\boldsymbol{k}_{b}^{\prime}$ is another wave vector of the fluctuating magnetic field. The data are taken from the Cluster observation in the foreshock region. The bispectrum is evaluated at various combinations of $\boldsymbol{k}_{b}$ and $\boldsymbol{k}_{n}$ using the resonance condition, and its distribution exhibits a peak at one particular combination of these two wave vectors.

The magnetic field and the electron data were used for the bispectral analysis for the following reason. There are various types of wave-wave interactions proposed in plasma physics, and one of them describes the interaction of an Alfvén wave (that is a fluctuation in the magnetic field) with a sound wave (that is a density fluctuation), generating another Alfvén wave at the resonant frequency and wave vector. The decay and the modulational instabilities belong to this family of interaction (Derby, 1978; Goldstein, 1978; Longtin and Sonnerup, 1986; Mjølhus, 1976; Spangler, 1999; Terasawa et al., 1986; Wong and Goldstein, 1986). The displayed example does not immediately imply an evidence of these instabilities in space plasma observation, but suggests that such an analysis can be performed both in the frequency and in the wave vector domain, which provides a means to study, either to prove or to disprove, these instabilities using spacecraft data. It is worth noting that Fig. 7 not only provides the direct evidence of wave-wave resonance in the spatial domain but also suggests that the parametric instability of Alfvén wave may be occurring in the foreshock region, in which an Alfvén wave collapses into another Alfvén wave and a density fluctuation (e.g., sound wave). However, more careful studies are needed to confirm the existence of parametric instability, for example, by investigating the bispectrum in the both frequency and wave vector domain; comparing the background condition such as the plasma beta with the theoretical predictions.

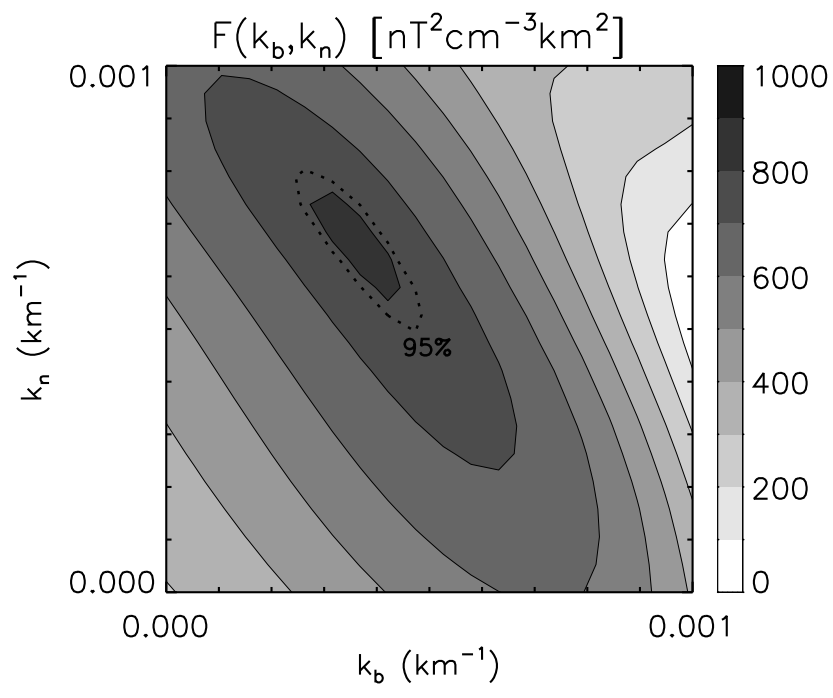

Fig. 7. Bispectrum using magnetic field and electron density data of Cluster in the foreshock under the resonance $k_{b}+k_{n}$, where $k_{b}$ and $k_{n}$ represent the wave number of the fluctuating magnetic field and electron density parallel to the mean magnetic field. The dotted line denotes the level of $95 \%$ confidence (Narita et al., 2008).

\section{Eigenvector analysis}

The CSD matrices of the state vectors are Hermitiansymmetric and they may be decomposed into a set of eigenvalues and eigenvectors, which have information about spatial structure of waves. Investigation of the eigenvectors of the CSD matrix represents provides another useful wave vector analysis tools. We present in this section two applications: the wave surveyor technique (Vogt et al., 2008) and the multi-point signal resonator (MSR) technique (Narita et al., 2010b). The former provides a wave vector identification method for the dominant wave components and the latter provides a high-resolution wave number spectrum.

\subsection{Wave surveyor technique}

The wave surveyor technique (Vogt et al., 2008) is a direct identification tool of wave dispersion relations in the sense that the wave vectors are computed directly as a function of the frequencies. The wave telescope technique, in contrast, provides the wave power in the frequency-wave vector domain and one has to search peaks of the energy distribution in the wave vector domain to identify the wave vectors of the dominant wave components, which is computationally demanding. The wave surveyor technique makes use of the eigenvectors of the CSD matrix, and it determines the wave vectors directly from the difference of wave phases measured at array-sensors, assuming a propagating plane wave. The eigenvectors of the CSD matrix are given as a set of complex numbers, and the phase $\theta_{i}$ at the $i$-th sensor should be equal or close to the phase of propagating plane wave in the 
observer's frame. We therefore minimize the deviation or difference between the ideal phases and the measured phases, i.e., minimize the following function

$Q(\boldsymbol{k}, \phi)=\sum_{i=1}^{L}\left[\theta_{i}-\boldsymbol{k} \cdot \boldsymbol{r}_{i}-\phi\right]^{2}$,

with respect to the wave vector $\boldsymbol{k}$ and the initial phase $\phi$. It can be shown that the wave vector can be directly obtained from the eigenvector phases as (Vogt et al., 2008):

$\boldsymbol{k}=\left(\sum_{i} \boldsymbol{r}_{i} \boldsymbol{r}_{i}^{t}\right)^{-1} \sum_{i} \theta_{i} \boldsymbol{r}_{i}$

Here $\boldsymbol{r}^{t}$ is the transposed vector of the sensor positions measured from the center of the sensor array. The position vectors satisfy the condition $\sum_{i} \boldsymbol{r}_{i}=0$. In the case of four sensors $(L=4)$ like the Cluster mission, the solution can be given as a linear combination of the reciprocal vectors of the spacecraft positions $\kappa_{i}$ (Neubauer and Glassmeier, 1990; Chanteur, 1998),

$\boldsymbol{k}(\omega)=\sum_{i=1}^{4} \theta_{i}(\omega) \boldsymbol{\kappa}_{i}$

This method directly determines the wave vector associated with the frequency $\omega$ is called the wave surveyor technique (Vogt et al., 2008). The wave surveyor technique gives a very similar result in the dispersion relation analysis to that of the wave telescope technique. Figure 8 displays the comparison of the dispersion relation for foreshock waves measured by Cluster. The dispersion relations in Fig. 8 agree with the theoretical prediction of ion beam instability (Gary, 1993), and therefore comfirm that the foreshock waves are driven by the backstreaming ion beams from the shock.

\subsection{MUSIC algorithm and MSR technique}

The eigenvectors of the CSD matrix are strictly orthogonal to one another, and this fact can be used for establishing another estimator of the wave number spectrum. The MUSIC algorithm (Multiple SIgnal Classification) was proposed by Schmidt (1986) on the assumption that the measured data contains signal and noise such that the CSD matrix can be decomposed into two parts. The state vector is therefore interpreted as a combination of the signal term and the noise term. Under this concept the CSD matrix is decomposed into the signal term and the noise term, too. The eigenvalues and eigenvectors of $\mathbf{R}$ are denoted by $\lambda_{1} \geq \lambda_{2} \geq \cdots \geq \lambda_{L}$ and $\boldsymbol{e}_{1}, \boldsymbol{e}_{2}, \cdots, \boldsymbol{e}_{L}$, respectively. For the noise part the eigenvalues are given as the "noise floor" (Haykin, 1991) such that $\lambda_{M+1}=\lambda_{M+2}=\cdots=\lambda_{L}=\sigma^{2}$. We split the eigenvectors of the CSD matrix $\mathbf{R}$ into two parts: the signal subspace $\mathbf{E}_{\mathrm{S}}=\left[\boldsymbol{e}_{1}, \boldsymbol{e}_{2}, \cdots, \boldsymbol{e}_{M}\right]$ and the noise subspace $\mathbf{E}_{n}=\left[\boldsymbol{e}_{M+1}, \boldsymbol{e}_{M+2}, \cdots, \boldsymbol{e}_{L}\right]$.
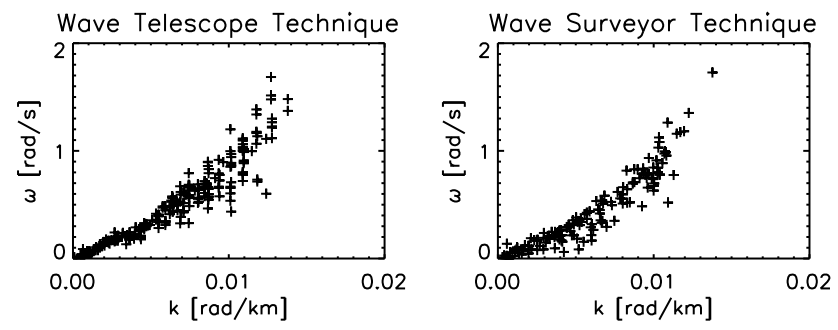

Fig. 8. Dispersion relation of foreshock waves measured by Cluster. Left panel displays the dispersion relation determined by the wave telescope technique (Narita et al., 2007), and right panel displays that determined by the wave surveyor technique (Vogt et al., 2008). Wave numbers are in the direction to the mean magnetic field and frequencies are given in the flow rest frame after the Dopper correction.

The power estimation in the MUSIC method is given as

$$
\begin{aligned}
P_{\text {MUSIC }} & =\frac{1}{\left|\boldsymbol{h}^{\dagger} \mathbf{E}_{n}\right|^{2}} \\
& =\frac{1}{\boldsymbol{h}^{\dagger} \mathbf{E}_{n} \mathbf{E}_{n}^{\dagger} \boldsymbol{h}},
\end{aligned}
$$

which makes use of the orthogonality between the steering vector $\boldsymbol{h}\left(\boldsymbol{k}_{i}\right)(i=1, \cdots, M)$ and the eigenvector for the noise part $\boldsymbol{e}_{j}(j=M+1, \cdots, L)$ :

$\boldsymbol{h}^{\dagger}\left(\boldsymbol{k}_{i}\right) \cdot \boldsymbol{e}_{j}=0$.

The MUSIC spectrum is also expressed using all eigenvectors as

$$
P_{\text {MUSIC }}=\frac{1}{\boldsymbol{h}^{\dagger} \mathbf{F L F} \boldsymbol{h}},
$$

where $\mathbf{F}$ is the eigenvector matrix of $\mathbf{R}$ sorted in descending order of the eigenvalues

$\mathbf{F}=\left[\mathbf{E}_{s} \mathbf{E}_{n}\right]=\left[\begin{array}{llllll}\boldsymbol{e}_{1} & \cdots & \boldsymbol{e}_{M} & \boldsymbol{e}_{M+1} & \cdots & \boldsymbol{e}_{L}\end{array}\right]$

The matrix $\mathbf{F}$ is an arrangement of the eigenvectors of the CSD matrix, placing the signal-associated eigenvectors on the left side in the matrix and the noise-associated eigenvectors on the right side. The matrix $\mathbf{L}$ is a diagonal matrix and is defined as

$$
\mathbf{L}=\operatorname{diag}(\underbrace{0, \cdots, 0}_{M}, \underbrace{1, \cdots, 1}_{L-M}) .
$$

The MUSIC algorithm is based on finding the eigenvectors associated with noise that are orthogonal to the steering vector with the signal wave vector. The spectrum estimated by the MUSIC algorithm uses the product of the noise eigenvectors and the steering vectors, and therefore the method gives the spectrum in the dimensionless unit. It should also 
be noted that the MUSIC algorithm requires that the number of signals must be known in the analysis to extract the set of the eigenvectors associated with noise. One method to determine the number of signals is to investigate the noise floor of the eigenvalues, for example, in a plot of eigenvalues in descending order (Haykin, 1991).

The problem that the number of signal sources must be known in the MUSIC algorithm was solved by Choi et al. (1993) by replacing the diagonal matrix $\mathbf{L}$ by $\Lambda^{-n}$ with

$\Lambda^{-n}=\operatorname{diag}\left(\left(\frac{\lambda_{1}}{\lambda_{L}}\right)^{-n},\left(\frac{\lambda_{2}}{\lambda_{L}}\right)^{-n}, \cdots,\left(\frac{\lambda_{L}}{\lambda_{L}}\right)^{-n}\right)$.

Here the power $-n$ is an adjustable parameter $(n=1,2, \cdots)$ in the analysis that controls the asymptotic behavior of the estimator such that the matrix $\Lambda^{-n}$ becomes $\mathbf{L}$ in the limit $n \rightarrow \infty$. In other words, replacing the matrix $\mathbf{L}$ by $\boldsymbol{\Lambda}^{-n}$ automatically selects the noise subspace of the CSD matrix $\mathbf{R}$. It should be noted that the procedure of the matrix replacement by $\boldsymbol{\Lambda}^{-n}$ does not stem from any mathematical theory guaranteeing better functionality of the technique, but it represents an intuitive picture of generalization of the matrix $\mathbf{L}$ to soften its sharp transition of the diagonal elements from zero to one. Therefore, other extensions or generalizations are possible for the MUSIC algorithm. Choi et al. (1993) found that even a small number of $n$ such as $n=2$ can successfully reproduce the MUSIC spectrum without knowing the number of signal sources. The spectrum using the extended MUSIC algorithm is given as

$$
P_{\mathrm{EM}}=\frac{1}{\boldsymbol{h}^{\dagger} \mathbf{F} \Lambda^{-n} \mathbf{F}^{\dagger} \boldsymbol{h}} \text {. }
$$

The spectrum $P_{\mathrm{EM}}$ is given in the dimensionless unit, but it may be used as a filter to Capon's spectrum. The MSR technique (Multi-point Signal Resonator) makes use of this notion to establish an estimator of high-resolution wave number spectra: We use Capon's estimator and obtain the power spectrum that exhibits the right value of the spectrum at the signal wave number; and we use additionally the extended MUSIC spectrum with a proper normalization as a dimensionless filter to enhance the signal-to-noise contrast of the Capon spectrum. The power spectrum in the MSR technique is therefore given as

$$
P_{\mathrm{MSR}}=\frac{1}{P_{\mathrm{EM} 0}} P_{\mathrm{EM}} P_{\mathrm{C}}
$$

Here the factor $1 / P_{\mathrm{EM} 0}$ denotes normalization of the extended MUSIC spectrum, and is a sum of the extendedMUSIC spectrum over the frequency-wave vector domain:

$$
P_{\mathrm{EM} 0}=\sum_{\omega, \boldsymbol{k}} P_{\mathrm{EM}}(\omega, \boldsymbol{k})
$$

The normalized, extended MUSIC spectrum $P_{\mathrm{EM}}(\omega, \boldsymbol{k}) / P_{\mathrm{EM} 0}$ serves as a filter that returns the value

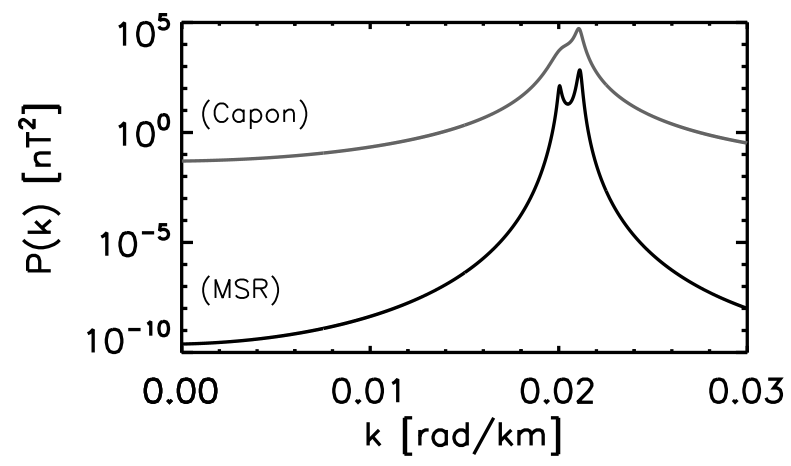

Fig. 9. Capon and MSR spectra for the synthetic data with two wave components that have similar or very close wavelengths (Narita et al., 2010b).

of almost one at the signal wave numbers and almost zero values otherwise, which enhances the quality of Capon's spectrum. Another merit of the MSR technique is that it can resolve waves with slightly different wavelengths or wave numbers. Figure 9 displays the spectral curves determined by the Capon and the MSR technique for a synthetic data set in which two wave components have close wave numbers to each other. The MSR spectrum can resolve two peaks at the signal wave numbers, whereas Capon's spectrum exhibits a peak with broadening. The peak at the smaller wave number in the MSR spectrum appears as a hump in the Capon spectrum, and identification of this peak is difficult. This example shows the ability of the MSR technique: much reduced background level; and high-resolution in the wave vector domain. The MSR technique can also be used for a measurement of a vector quantity such as the magnetic field. It is also possible to set the divergence-free condition as an additional constraint. Details of the MSR technique are discussed in Narita et al. (2010b).

\section{Conclusions}

"Cluster is more than just a four spacecraft mission" so initiates the paper presenting the first result of the wave telescope technique using Cluster data (Glassmeier et al., 2001). The motivation to analyze data in the wave vector domain despite the small number of measurement points has been worked out and is still being developed for more than two decades. Fluctuation energy, helicity, and higher order moments are very important quantities characterizing properties of plasma turbulence, and with Cluster one can evaluate them in the 3-D wave vector domain. Taylor's hypothesis or axisymmetry around the mean magnetic field are not any more necessary assumptions in interpreting Cluster data, and furthermore, Cluster provides the opportunity to verify these assumptions. Although the range of wave vectors is rather narrow because it is determined by the inter-spacecraft separation and the tetrahedral configuration, Cluster offers various 
separation and configuration phases in its operation, ranging from $100 \mathrm{~km}$ to $10000 \mathrm{~km}$ separation. It would be interesting to study turbulent fluctuations in the solar wind and the magnetosphere using the methods presented in this paper, to see how spatial properties change from a large scale to a small scale. Also, the analysis methods can be applied to the plasma data, in particular, the flow velocity. The wave vector analysis using both the magnetic field and the flow velocity data would provide more complete information about the nature of plasma turbulence.

Acknowledgements. This work was financially supported by Bundesministerium für Wirtschaft und Technologie and Deutsches Zentrum für Luft- und Raumfahrt, Germany, under contract 50 OC 0901.

Edited by: B. Tsurutani

Reviewed by: S. P. Gary and another anonymous referee

\section{References}

Balogh, A., Carr, C. M., Acuña, M. H., Dunlop, M. W., Beek, T. J., Brown, P., Fornacon, K.-H., Georgescu, E., Glassmeier, K.H., Harris, J., Musmann, G., Oddy, T., and Schwingenschuh, K.: The Cluster Magnetic Field Investigation: overview of in-flight performance and initial results, Ann. Geophys., 19, 1207-1217, doi:10.5194/angeo-19-1207-2001, 2001.

Biskamp, D.: Magnetohydrodynamic Turbulence, Cambridge University Press, Cambridge, 2003.

Capon, J.: High resolution frequency-wavenumber spectrum analysis, Proc. IEEE, 57, 1408-1418, 1969.

Carbone, V., Malara, F., and Veltri, P.: A model for the threedimensional magnetic field correlation spectra of low-frequency solar wind fluctuations during Alfvénic periods, J. Geophys. Res., 100, 1763-1778, 1995.

Chanteur, G.: Spatial interpolation for four spacecraft: Theory, in Analysis Methods for Multi-Spacecraft Data, G. Paschmann and P. Daly (eds.), ISSI Scientific Reports Series, ESA/ISSI, 1, 349370, 1998.

Choi, J., Song, I., and Kim, H. M.: On estimating the direction of arrival when the number of signal sources is unknown, Signal Process., 34, 193-205, 1993.

Coleman Jr., P. J.: Turbulence, viscosity, and dissipation in the solar-wind plasma, Astrophys. J., 153, 371-388, 1968.

Constantinescu, O. D., Glassmeier, K.-H., Motschmann, U., Treumann, R. A., Fornaçon, K.-H., and Fränz, M.: Plasma wave source location using CLUSTER as a spherical wave telescope, J. Geophys. Res., 111, A09221, doi:10.1029/2005JA011550, 2006.

Constantinescu, O. D., Glassmeier, K.-H., Décréau, P. M. E., Fränz, M., and Fornaçon, K.-H.: Low frequency wave sources in the outer magnetosphere, magnetosheath, and near Earth solar wind, Ann. Geophys., 25, 2217-2228, doi:10.5194/angeo-25-22172007, 2007.

Constantinescu, O. D.: Wave sources and structures in the Earth's magnetosheath and adjacent regions, Ph.D. thesis, Copernicus GmbH, Katlenburg-Lindau, 2007.
Dasso, S., Milano, L. J., Matthaeus, W. H., and Smith, C. W.: Anisotropy in fast and slow solar wind fluctuations, Astrophys. J., 635, L181-L184, 2005.

Derby Jr., N. F.: Modulational instability of finite-amplitude, circularly polarized Alfvén waves, Astrophys. J., 224, 1013-1016, 1978.

Escoubet, C. P., Fehringer, M., and Goldstein, M.: Introduction The Cluster mission, Ann. Geophys., 19, 1197-1200, doi:10.5194/angeo-19-1197-2001, 2001.

Gary, S. P.: Theory of space plasma microinstabilities. Cambridge Atmos, Space Science Series, Cambridge, 1993.

Glassmeier, K.-H., Coates, A. J., Acuña, M. H., Goldstein, M. L., Johnstone, A. D., Neubauer, F. M., and Rème, H.: Spectral characteristics of low-frequency plasma turbulence upstream of Comet P/Halley, J. Geophys. Res., 94, 37-48, 1989.

Glassmeier, K.-H., Motschmann, U., Dunlop, M., Balogh, A., Acuña, M. H., Carr, C., Musmann, G., Fornaçon, K.-H., Schweda, K., Vogt, J., Georgescu, E., and Buchert, S.: Cluster as a wave telescope - first results from the fluxgate magnetometer, Ann. Geophys., 19, 1439-1447, doi:10.5194/angeo-19-14392001, 2001 (correction in 21, p. 1071, 2003).

Goldstein, M. L.: An instability of finite amplitude circularly polarized Alfvén waves, Astrophys. J., 219, 700-704, 1978.

Harjes, H. P. and Henger, M.: Array Seismologie, Zeitschrift f. Geophys., 39, 865-905, 1973.

Haykin, S.: Adaptive filter theory, 2nd edn., Prentice Hall information and system science series, Prentice-Hall Inc., New Jersey, USA, 1991.

Longtin, M. and Sonnerup, B.: Modulational instability of circularly polarized Alfvén waves, J. Geophys. Res., 91, 798-801, 1986.

Marsch, E. and Tu, C.-Y.: On the radial evolution of MHD turbulence in the inner heliosphere, J. Geophys. Res., 95, 8211-8229, 1990.

Marsch, E.: MHD turbulence in the solar wind, Physics of the Inner Heliosphere, Vol. II, edited by: Schwenn, R. and Marsch, E., Springer Verlag, Heidelberg, 159-241, 1995.

Matthaeus, W. H. and Goldstein, M. L.: Measurement of the rugged invariants of magnetohydrodynamic turbulence in the solar wind, J. Geophys. Res., 87, 6011-6028, 1982.

Matthaeus, W. H., Goldstein, M. L., and Smith, C.: Evaluation of magnetic helicity in homogeneous turbulence, Phys. Rev. Lett., 48, 1256-1259, 1982.

Matthaeus, W. H., Goldstein, M. L., and Roberts, D. A.: Evidence for the presence of quasi-two-dimensional nearly incompressible fluctuations in the solar wind, J. Geophys. Res., 95, 2067320683, 1990.

Mjølhus, E.: On the modulational instability of hydromagnetic waves parallel to the magnetic field, J. Plasma Phys., 16, 321334, 1976.

Motschmann, U., Woodward, T. I., Glassmeier, K.-H., Southwood, D. J., and Pinçon, J. L.: Wavelength and direction filtering by magnetic measurements at satellite arrays: generalized minimum variance analysis, J. Geophys. Res., 101, 4961-4965, 1996.

Musmann, G., Beinroth, H. J., Denskat, U., Hente, B., Theile, B., and Neubauer, F.: Proposal for a plasma wave array experiment to be flown on the ESA Spacelab, European Space Agency, submitted, 1974. 
Narita, Y., Glassmeier, K.-H., and Treumann, R. A.: Magnetic turbulence spectra in the high-beta plasma upstream of the terrestrial bow shock, Phys. Rev. Lett., 97, 191101, 2006.

Narita, Y., Glassmeier, K.-H., Fränz, M., Nariyuki, Y., and Hada, T.: Observations of linear and nonlinear processes in the foreshock wave evolution, Nonlin. Processes Geophys., 14, 361-371, doi:10.5194/npg-14-361-2007, 2007.

Narita, Y., Glassmeier, K.-H., Décréau, P. M. E., Hada, T., Motschmann, U., and Nariyuki, Y.: Evaluation of bispectrum in the wave number domain based on multi-point measurements, Ann. Geophys., 26, 3389-3393, doi:10.5194/angeo-26-33892008, 2008.

Narita, Y. and Glassmeier, K.-H.: Spatial aliasing and distortion of energy distribution in the wave vector domain under multi-spacecraft measurements, Ann. Geophys., 27, 3031-3042, doi:10.5194/angeo-27-3031-2009, 2009a.

Narita, Y., Kleindienst, G., and Glassmeier, K.-H.: Evaluation of magnetic helicity density in the wave number domain using multi-point measurements in space, Ann. Geophys., 27, 39673976, doi:10.5194/angeo-27-3967-2009, 2009. b.

Narita, Y., Sahraoui, F., Goldstein, M. L., and Glassmeier, K.-H.: Magnetic energy distribution in the four-dimensional frequency and wave vector domain in the solar wind, J. Geophys. Res., 115, A04101, doi:10.1029/2009JA014742, 2010a.

Narita, Y., Glassmeier, K.-H., and Motschmann, U.: Highresolution wave number spectrum using multi-point measurements in space - the multi-point signal resonator (MSR) technique, Ann. Geophys., in review, 2010b.

Narita, Y., Glassmeier, K.-H., Goldstein, M. L., and Sahraoui, F.: Wave-vector dependence of magnetic-turbulence spectra in the solar wind, Phys. Rev. Lett., 104, 171101, doi:10.1103/PhysRevLett.104.171101, 2010c.

Narita, Y. and Glassmeier, K.-H.: Anisotropy evolution of magnetic field fluctuation through the bow shock, Earth Planets Space, 62, e1-e4, 2010d.

Neubauer, F. M. and Glassmeier, K.-H.: Use of an array of satellites as a wave telescope, J. Geophys. Res., 95, 19115-19122, 1990.

Pinçon, J. L. and Lefeuvre, F.: Local characterization of homogeneous turbulence in a space plasma from simultaneous measurement of field components at several points in space, J. Geophys. Res., 96, 1789-1802, 1991.

Pinçon, J.-L. and Motschmann, U.: Multi-Spacecraft Filtering: General Framework, Analysis methods for multi-spacecraft data, edited by: Paschmann, G. and Daly, P. W., ISSI Scientific Report, SR-001, Bern, 1998.

Pinçon, J.-L. and Glassmeier, K.-H.: Multi-spacecraft methods of wave field characterization, Multi-Spacecraft Analysis Methods Revisited, edited by: Paschmann, G. and Daly, P. W., ISSI Scientific Report, SR-008, ISSI/ESA, Noordwijk, 2008.
Plaschke, F., Glassmeier, K.-H., Constantinescu, O. D., Mann, I. R., Milling, D. K., Motschmann, U., and Rae, I. J.: Statistical analysis of ground based magnetic field measurements with the field line resonance detector, Ann. Geophys., 26, 3477-3489, doi:10.5194/angeo-26-3477-2008, 2008.

Podesta, J. J., Roberts, D. A., and Goldstein, M. L.: Spectral exponents of kinetic and magnetic energy spectra in solar wind turbulence, Astrophys. J., 664, 543-548, 2007.

Sahraoui, F., Pinçon, J. L., Belmont, G., Rezeau, L., CornilleauWehrlin, N., Robert, P., Mellul, L., Bosqued, J. M., Balogh, A., Canu, P., and Chanteur, G.: ULF wave identification in the magnetosheath: The k-filtering technique applied to Cluster II data, J. Geophys. Res., 108, SMP1-1, 1335, doi:10.1029/2002JA009587, 2003 (correction in 109, A04222, doi10.1029/2004JA010469, 2004).

Sahraoui, F., Belmont, G., Rezeau, L., Cornilleau-Wehrlin, N., Pinon, J. L., and Balogh, A.: Anisotropic turbulent spectra in the terrestrial magnetosheath as seen by the Cluster spacecraft, Phys. Rev. Lett., 96, 075002, PMID:16606099, 2006.

Sahraoui, F., Belmont, G., Goldstein M., and Rezeau, L.: Limitations of multi-spacecraft data techniques in measuring wavenumber spectra of space plasma turbulence, J. Geophys. Res., in press, doi:10.1029/2009JA014724, 2010.

Schmidt, R. O.: Multiple emitter location and signal parameter estimation, IEEE T. Antenn., Propag., AP-34, 276-280, 1986.

Spangler, S. R.: Two-dimensional magnetohydrodynamics and interstellar plasma turbulence, Astrophys. J., 522, 879-896, 1999.

Stix, T. H.: Waves in plasmas, Springer-Verlag, New York, 1992.

Taylor, G. I.: The spectrum of turbulence, P. Roy. Soc. Lond. A, 164, 476-490, 1938.

Terasawa, T., Hoshino, M., Sakai, J.-I., and Hada, T.: Decay instability of finite-amplitude circularly polarized Alfvén waves: A numerical simulation of stimulated brillouin scattering, J. Geophys. Res., 91, 4171-4187, 1986.

Tu, C.-Y. and Marsch, E.: MHD structures, waves, and turbulence in the solar wind: observations and theories, Space Sci. Rev., 73, 1-210, 1995.

Vogt, J., Narita, Y., and Constantinescu, O. D.: The wave surveyor technique for fast plasma wave detection in multi-spacecraft data, Ann. Geophys., 26, 1699-1710, doi:10.5194/angeo-261699-2008, 2008.

Wong, H. K. and Goldstein, M. L.: Parametric instabilities of circularly polarized Alfvén waves including dispersion, J. Geophys. Res., 91, 5617-5628, 1986. 